\title{
The Relation of Urinary 8-OHdG, A Marker of Oxidative Stress to DNA, and Clinical Outcomes for Ischemic Stroke
}

\author{
Hideto Nakajima ${ }^{1,2 *}$, Ki-ichi Unoda ${ }^{1,2}$, Takumi Ito ${ }^{1}$, Haruko Kitaoka ${ }^{1}$, Fumiharu Kimura ${ }^{2}$, and \\ Toshiaki Hanafusa ${ }^{2}$
}

\author{
${ }^{1}$ Department of Internal Medicine, Seikeikai Hospital, Sakai 590-0024, Japan \\ ${ }^{2}$ Department of Internal Medicine I, Osaka Medical College, Takatsuki 569-8686, Japan
}

\begin{abstract}
Background: Oxidative stress/free radical generation after ischemic stroke contributes to neuronal cell injury. We evaluated the utility of an oxidative stress marker, urinary 8-hydroxy-2-deoxyguanosine (8-OHdG), to demonstrate an association between the changes of $8-\mathrm{OHdG}$ and outcomes after acute ischemic stroke.

Methods: We enrolled 44 patients (26 males and 18 females) who visited our hospital due to acute ischemic stroke. Urine was collected on admission and on Days 7, and 8-OHdG was measured by ELISA. The relationships between 8-OHdG levels, stroke subtypes, and clinical outcomes based on the NIHSS and modified Rankin Scale (mRS) upon discharge was evaluated.
\end{abstract}

Results: In the overall cohort, the mean urinary level of 8-OHdG on Day 7 was increased than that on Day 0. The 8OHdG levels on Day 0 were not different between patients with poor and good outcomes. However, an increasing rate from Day 0 to $7(\Delta 8-\mathrm{OHdG})$ in stroke patients with a poor outcome $(\mathrm{mRS} \geq 3)$ was significantly higher than those with a good outcome $(\mathrm{mRS} \leq 2)(2.54$ vs $39.44, \mathrm{p}=0.004)$.

Conclusions: The biochemical changes related to $8-\mathrm{OHdG}$ and oxidative stress may be considered a marker of ischemic brain injury and clinical outcome of ischemic stroke.

Keywords: Ischemic stroke, Oxidative stress, 8-OHdG, Clinical outcome, Radical scavenger, Edaravone.

\section{INTRODUCTION}

Oxidative stress is one of the mechanisms involved in ischemic brain injury induced by ischemia-reperfusion [1,2]. In addition to the direct cytotoxic effects of lipid peroxidation, oxidative DNA damage occurs immediately after ischemia-reperfusion and leads to ischemic brain injury, as reflected by neural death and brain edema [3-7]. Thus, the burst of free radicals is thought to be associated with stroke severity. However, it remains uncertain how to evaluate the association between increased oxidative stress and functional outcomes of patients. In the presence of oxidative stress, reactive oxygen species, including superoxide anion radicals, hydrogen peroxide, and hydroxyl radicals, can cause oxidative damage to lipids, proteins, and nucleic acids [8]. During oxidative stress, DNA is constantly being damaged and oxidatively modified. Nuclear and mitochondrial DNA in tissues and blood lymphocytes are typical sites of oxidation damage [8].

The oxidatively modified product 8-hydroxy-2deoxyguanosine (8-OHdG) is one of the predominant forms of free radical-induced lesions of DNA. 8-OHdG can be detected in human tissues or blood samples. Because oxidative

\footnotetext{
*Address correspondence to this author at the Department of Internal Medicine Seikeikai Hospital, 4-2-10 Koryonakamachi, Sakai, Osaka 590-0024 (Japan); Tel.: +81 72251 8199; Fax: +81 72259 3691;

E-mail: in1045@poh.osaka-med.ac.jp
}

DNA lesions, i.e., oxidized nucleosides and bases, are reasonably water soluble and excreted into the urine without being further metabolized, urinary $8-\mathrm{OHdG}$ is considered an important biomarker of generalized and cellular oxidative stress [9]. In fact, urinary $8-\mathrm{OHdG}$ levels have been most frequently measured to indicate the extent of oxidative damage, since it is a non-invasive and technically less involved procedure [10].

Edaravone (3-methyl-1-phenyl-2-pyrazolin-5-one, Mitsubishi Pharma Corp), a novel radical scavenger, has been shown to inhibit lipid peroxidation and vascular endothelial cell injury and ameliorate brain edema, tissue injury, delayed neuronal death, and neurological deficits [11-13]. Edaravone is the first neuroprotective agent to be approved for clinical use in Japan [14]. The effect of edaravone on ischemic processes has been investigated experimentally, but no data is available on its effects on oxidative stress markers in the human ischemic brain.

In the present study, we examined oxidative stress in patients with acute ischemic stroke by determining the changes in urinary $8-\mathrm{OHdG}$, a maker of oxidative damage. We evaluated the relationships between $8-\mathrm{OHdG}$ levels, stroke subtypes, and clinical outcomes based on the National Institutes of Health Stroke Scale (NIHSS) and the modified Rankin Scale (mRS) at discharge. The effect of edaravone on oxidative damage was also examined. 


\section{MATERIALS AND METHODOLOGY}

\subsection{Patients}

Forty-four patients (males: 26, females: 18; age: 29-92 years, mean \pm SD: $71.3 \pm 10.9$ years) with acute cerebral infarction from July 2007 to February 2008 were enrolled in this study in the Department of Internal Medicine at Seikeikai Hospital, Sakai, Osaka. All patients underwent an MRI examination immediately at admission, and diagnosis was based on clinical findings. An NIHSS score was assigned both at admission and upon discharge to determine neurological deficits [15]. The mRS was also used to assess their clinical outcomes at discharge [16]. Conventional vascular risk factors (hypertension, diabetes mellitus, hyperlipidemia), atrial fibrillation, body mass index (BMI), and previous stroke were evaluated. Patients whose data could not be evaluated at the time of stroke onset and those with hemorrhagic infarction were excluded from this study. Stroke subtypes were defined according to the NINDS-III classification system [17]. Of the 44 patients, $9(20.5 \%)$ had lacunar, $22(50 \%)$ had atherothrombotic, and $13(29.5 \%)$ had cardioembolic infarctions. Of these, 34 patients were treated with edaravone (3-methyl-1-phenyl-2-pyrazolin-5-one, a free-radical scavenge, Mitsubishi Pharma Corp) in addition to routine treatment within $24 \mathrm{~h}$ of onset of symptoms ( 60 $\mathrm{mg}$ /day for 14 days). The remaining patients did not receive edaravone, because this medical agent is relative contraindications for patients with chronic renal failure and has been approved for the treatment of acute ischemic stroke within 24 hours after onset. All patients with lacunar and atherothrombotic infarctions received sodium ozagrel, a thromboxane A2 synthetase inhibitor, intravenously $(160 \mathrm{mg} /$ day for 10-14 days), and 35 patients received glycerol (250-500 $\mathrm{ml} /$ day for 3-7 days). There was no patient with thrombolytic therapy in this study. This study received institutional review board approval and informed consent was obtained according to the Declaration of Helsinki.

\subsection{Measurement of Urinary 8-OHdG}

Urine samples were collected on admission (Day 0 ) and 7 days after admission (Days 7). Urinary 8-OHdG concentrations were measured using an enzyme-linked immunosorbent assay (8-Hydroxydeoxyguanosine Check; Japan Institute for the Control of Aging, Shizuoka, Japan) [18]. Results were expressed as ratios to the creatinine contents (per $\mathrm{mg} \mathrm{Cr}$ ) measured in the same samples.

Moreover, we evaluated the rate of change of oxidative $\operatorname{stress}(\Delta 8$-OHdG) as: $\Delta 8$-OHdG $(\%)=(8-\mathrm{OHdG}$ on Day 7 8-OHdG on Day 0)/8-OHdG on Day 0.

\subsection{Statistical Analysis}

Results are given as means \pm SEs. Categorical variables were compared using the $\chi^{2}$ test and continuous variables were compared using the Mann-Whitney test or ANOVA, followed by Scheffe's post hoc test. Significance levels were set at $P<0.05$.

\section{RESULTS}

3.1. Urinary 8-OHdG and Relationships to Subtypes of Ischemic Stroke and Risk Factors for Arteriosclerosis

Patients clinical profiles according to ischemic stroke subtypes are summarized in Table $\mathbf{1}$. There were no significant differences with respect to age or history of previous stroke among the 3 groups. Prevalence of auricular fibrillation was the highest among those with cardiac embolism. Regarding risk factors for arteriosclerosis, prevalence of hypertension was the highest in patients with lacunar infarction, and incidence of diabetes mellitus and hyperlipidemia were the highest in those with atherothrombotic infarctions. The number of arteriosclerosis risk factors was also higher in lacunar and artherothrombotic infarction groups compared to cardioembolic infarction group. The cardioembolic infarction group had a shorter time from onset to arrival, higher baseline NIHSS, and longer length of stay compared to the other 2 groups. There were no significant differences in 8OHdG concentrations on Day 0 among the 3 groups, and 8OHdG concentrations increased on Day 7 in all groups (Table 1)

We investigated the relationship between $\Delta 8-\mathrm{OHdG}$ and stroke subtype, lacunar, atherothrombotic, and cardioembolic infarction. As shown in Fig (1), the increase in $\Delta 8$-OHdG in the atherothrombotic infarction group was significantly greater than that in the lacunar infarction group (33.8 vs 6.3, $\mathrm{p}=0.048)$. It was remarkable that $\Delta 8$-OHdG in the atherothrombotic infarction group tended to be increased than that in the cardioembolic infarction group in spite of smaller volume of infarction for atherothrombotic infarction group. As shown in Table 1, patients with atherothrombotic infarction had more risk factors for arteriosclerosis than other groups. Also, half of patients with atherothrombotic infarction showed poor outcome ( $\mathrm{mRS} \geq 3$ ). Since it was assumed that these risk factors might influence on a production of the oxidative stress, we investigated whether risk factors for arteriosclerosis, hyperetension, diabetes mellitus and hyperlipidemia, and body mass index (BMI) would involve the $\Delta 8$ OHdG. As results, patients with diabetes mellitus, hyperlipidemia, or high BMI showed the increased $8-\mathrm{OHdG}(\mathrm{p}=$ $0.047, \mathrm{p}=0.037, \mathrm{p}=0.033$, Fig 2). These findings suggest

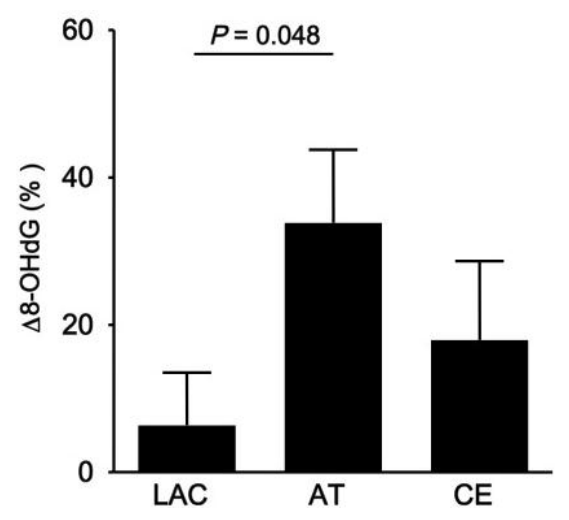

Fig. (1). $\triangle 8-\mathrm{OHdG}$ according to the subtypes of ischemic stroke, lacunar (LAC), atherothrombotic (AT), and cardioembolic (CE) infarction groups. $\triangle 8-\mathrm{OHdG}$ for AT was significantly increased compared to that for LAC. 
Table 1. Characteristics of Patients Recruited in This Study

\begin{tabular}{|c|c|c|c|c|}
\hline & Lacunar & Atherothrombotic & Cardioembolic & $P$ value \\
\hline Age & 74 & 69 & 74 & 0.298 \\
\hline Male/female & $5 / 4$ & $8 / 14$ & $7 / 6$ & 0.826 \\
\hline Previous stroke, \% (n) & $44(4)$ & $18(4)$ & $8(1)$ & 0.103 \\
\hline \multicolumn{5}{|l|}{ Risk factors for arteriosclerosis } \\
\hline Hypertension, \% (n) & $100(9)$ & $73(16)$ & $15(2)$ & $0.001>$ \\
\hline Diabetes mellitus, \% (n) & $0(0)$ & $36(8)$ & $8(1)$ & 0.029 \\
\hline Hyperlipidemia, \% (n) & $22(2)$ & $50(11)$ & $23(3)$ & 0.171 \\
\hline Baseline NIHSS & 4 & 7 & 19 & 0.003 \\
\hline $\mathrm{mRS}$ at discharge & 1.9 & 2.6 & 3.7 & 0.065 \\
\hline$m R S \geq 3, \%(n)$ & $33.3(3)$ & $50.0(11)$ & $84.6(11)$ & 0.038 \\
\hline Length of stay (days) & 28 & 29 & 39 & 0.028 \\
\hline \multicolumn{5}{|c|}{ 8-OHdG concentration (ng/mgCr) } \\
\hline Day 0 & $15.8 \pm 6.9$ & $12.8 \pm 11.7$ & $11.8 \pm 5.6$ & 0.128 \\
\hline Day 7 & $16.1 \pm 5.1$ & $16.2 \pm 15.5$ & $13.0 \pm 5.0$ & 0.272 \\
\hline
\end{tabular}
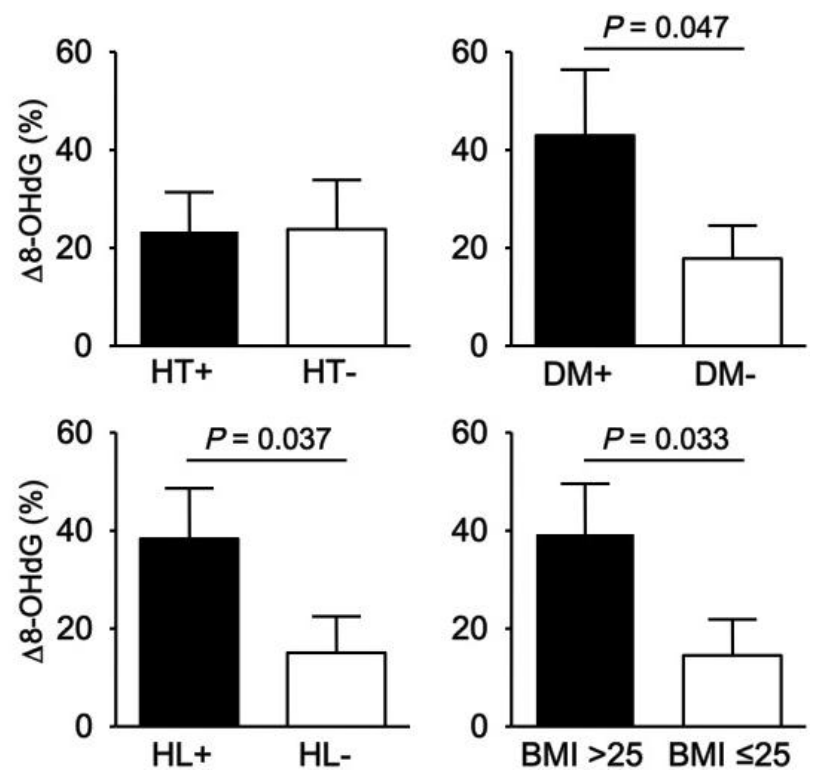

Fig. (2). Relationship between $\Delta 8-\mathrm{OHdG}$ and risk factors for arteriosclerosis. Stroke patients with diabetes mellitus DM), hyperlipidemia (HL), or high body mass index (BMI) showed the increased 8-OHdG. HT; hypertension.

that more oxidative stress would be generated in stroke patients with more risk factors for arteriosclerosis, reflecting poor outcome.

\subsection{Relationship Between Clinical Outcome and Urinary 8-OHdG}

Next, we investigated whether urinary $8-\mathrm{OHdG}$ concentrations reflected the severity and outcome of ischemic 
stroke. When comparing patients with a poor outcome (mRS $\geq 3$ ) and a good outcome ( $\mathrm{mRS} \leq 2)$, no significant differences were observed for age, history of previous stroke and atrial fibrillation, and prevalence of risk factors for arteriosclerosis (Table 2). Among patients with cardioembolic infarctions, significantly more number of patients had a poor outcome. Accordingly, NIHSS scores at admission were higher and the time from onset to arrival was shorter in patients with a poor outcome. There was no significant difference in 8OHdG concentrations on Day 0 between patients with poor and good outcomes, and 8-OHdG concentrations increased on Day 7 in both groups (Table 2). As shown in Fig (3), patients with a poor outcome had a significantly increased $\Delta 8$ OHdG compared to those with a good outcome (2.54 vs $39.44, \mathrm{p}=0.004)$. These findings suggested that the biochemical changes related to $8-\mathrm{OHdG}$ and oxidative stress may be considered a marker of ischemic brain injury and clinical outcome of ischemic stroke.

\subsection{Effect of Edaravone Treatment on Urinary $\Delta 8$ - OHdG}

On comparing the patients treated with or without edaravone, no significant differences were observed for age, stroke subtypes, and risk factors for arteriosclerosis (Table 3).
Based on NIHSS scores, patients who received edaravone tended to be classified as more severe at admission compared to those who did not receive edaravone (11.1 vs 6.1 ); however, both showed equivalent $\mathrm{mRS}$ scores at discharge $(2.5$ vs 2.7). Although there was no significant difference in $\Delta 8$ OHdG levels between patients treated with or without edaravone, $\Delta 8-\mathrm{OHdG}$ in patients who received edaravone tended to be reduced compared to those who did not receive edaravone within the atherothrombotic infarction group (23.5 vs 46.5, $\mathrm{p}=0.433$, Fig 4).

\section{DISCUSSION}

In order to evaluate ischemic brain damage, numerous biomarkers related to oxidative stress, such as nitric oxide, matrix metalloproteinase-9, S100 $\beta$, and oxidized LDL, have been investigated [19-22]. The main finding of our study is the demonstration of an association between urinary 8$\mathrm{OHdG}$ and ischemic stroke. The present study provides evidence that $\Delta 8-\mathrm{OHdG}$, specifically the rate of change of urinary $8-\mathrm{OHdG}$, may be considered a marker of tissue injury in brain ischemia. In fact, $\mathrm{mRS}$ scores (i.e., clinical outcomes at discharge) showed a significant correlation with $\Delta 8$ OHdG (Fig 3). These findings suggest that $\Delta 8-\mathrm{OHdG}$ may act as a mediator during the evolution of ischemic damage.

Table 2. Demographics and 8-OHdG Concentrations of Patients with Good and Poor Outcomes

\begin{tabular}{|c|c|c|c|}
\hline & Good $(\operatorname{mRS} \leq 2)$ & Poor $(m R S \geq 3)$ & $P$ value \\
\hline Male/female & $12: 07$ & $13: 12$ & 0.066 \\
\hline \multicolumn{4}{|l|}{ Stroke subtypes } \\
\hline Previous stroke, \% (n) & $21(4)$ & $20(5)$ & 1.000 \\
\hline Atrial fibrillation, \% (n) & $26(5)$ & $44(11)$ & 0.344 \\
\hline \multicolumn{4}{|l|}{ Risk factors for arteriosclerosis } \\
\hline Hypertension, \% (n) & $68(13)$ & $60(15)$ & 0.753 \\
\hline Number of risk factors & 1.3 & 1.2 & 0.697 \\
\hline Baseline NIHSS & 3.7 & 14.1 & 0.001 \\
\hline Time from onset to arrival (h) & 15.8 & 12.3 & 0.177 \\
\hline Edaravone, \% (n) & $74(14)$ & $80(20)$ & \\
\hline \multicolumn{4}{|c|}{ 8-OHdG concentration ( $\mathrm{ng} / \mathrm{mgCr})$} \\
\hline Day 0 & $12.1 \pm 6.3$ & $13.3 \pm 11.2$ & 0.538 \\
\hline Day 7 & $12.8 \pm 6.1$ & $17.1 \pm 14.1$ & 0.205 \\
\hline
\end{tabular}

LAC; lacunar infarction, AT; atherothrombotic infarction, CE; cardioembolic infarction 


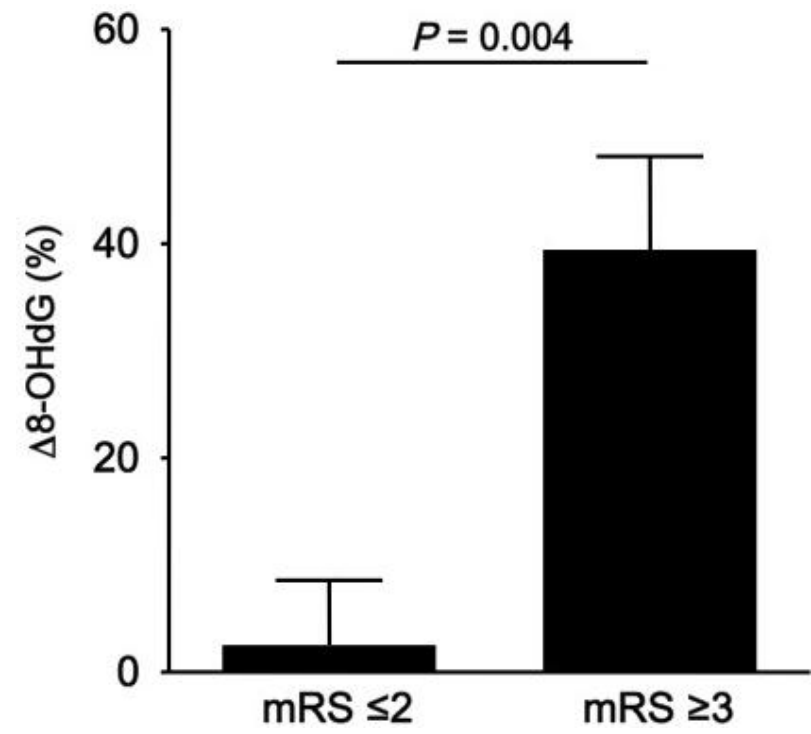

Fig. (3). Relationship between $\Delta 8-\mathrm{OHdG}$ and $\mathrm{mRS}$ scores at discharge. Patients with a poor outcome (mRS $3 \leq$ ) had a significantly increased $\Delta 8$-OHdG compared to those with a good outcome (mRS $2 \geq)$.

In all 3 subtypes of ischemic stroke, lacunar, atherothrombotic, and cardioembolic infarctions, urinary 8-OHdG levels increased on Day 7. When we compared the rate of change of urinary $8-\mathrm{OHdG}(\Delta 8-\mathrm{OHdG}), \Delta 8-\mathrm{OHdG}$ was significantly higher in patients in the atherothrombotic infarction group than that of patients in the lacunar infarction group (Fig 1). Moreover, $\Delta 8-\mathrm{OHdG}$ of patients in the lacunar infarction group tended to be lower than that of patients in the cardioembolic infarction group, suggesting that a smaller infarction volume can result in lesser amounts of oxidative stress. However, the questions arise as to why $\Delta 8$ $\mathrm{OHdG}$ increased more in the atherothrombotic infarction than in the cardioembolic infarction group.

Numerous studies have shown that urinary $8-\mathrm{OHdG}$ is not only a biomarker of oxidative stress but also a possible risk factor for cancer, coronary heart disease, diabetes, and atherosclerosis [23,24]. For example, elevated levels of urinary $8-\mathrm{OHdG}$ have been detected in patients with diabetes mellitus or hypertension [25]. In human atherosclerotic plaques, increased amounts of oxidatively modified DNA and 8-OHdG are present [26]. Thus, DNA oxidative stress may readily occur in patients with these conditions. In this study, the atherothrombotic infarction group had more number of risk factors for arteriosclerosis, such as hypertension, diabetes mellitus, and hyperlipidemia, and the cardioembolic group had less number of risk factors than the other groups (Table 1). In addition, the risk factors for arteriosclerosis such as diabetes mellitus, hyperlipidemia, and BMI influence on a production of the oxidative stress (Fig 2). Thus, patients with atherothrombotic infarctions may have some background conditions for which $8-\mathrm{OHdG}$ is easily generated, and more increased oxidative damage may cause poor outcome comparable to that of patients with cardioembolic infarctions, despite having the less infarction volume.

There is increasing evidence for the contributions of oxidative stress to ischemic brain damage in primary and sec- ondary pathophysiological mechanisms $[1,2]$. In addition to the direct cytotoxic effects of lipid peroxidation and oxidative DNA damage that occurs immediately after ischemic injury, the burst of free radicals also induces formation of inflammatory mediators through redox-mediated signaling pathways, leading to post-ischemia/reperfusion inflammatory injury $[4,6]$. Therefore, the control of these 2 impacts of oxidative damage is important to achieve neuroprotection.

Recent studies demonstrated that edaravone provided neuroprotection in the integrated processes of oxidative stress-induced injury in ischemic stroke [27,28]. Edaravone has antioxidant properties that result in suppressing lipid peroxidation products and oxidative DNA damage, as well as anti-inflammatory effects that result in reduced iNOS production [29-33]. Further studies by using animal model suggested that a combination therapy with edaravon and tissue plasminogen activator (rt-PA) could provide important therapeutic benefits for acute stroke patients, not only in reducing the infarct size but also in minimizing the catastrophic hemorrhagic transformation. In these studies, edaravone suppressed matrix metalloproteinase-9 (MMP-9) upreguration and hemorrhagic transformation by scavenging rt-PAinduced reactive oxidative stress derived from ische$\mathrm{mia} /$ reperfusion injury $[34,35]$.

As the present study was not a randomized trial, there could have been a bias in the selection of patients for edaravone use. However, although patients who received edaravone tended to be classified as more severe based on NIHSS scores at admission than those who did not receive edaravone, both groups showed equivalent $\mathrm{mRS}$ scores at discharge (Table 3). Furthermore, $\Delta 8-\mathrm{OHdG}$ levels in patients who received edaravone tended to be reduced compared to those who did not receive edaravone within the atherothrombotic infarction group (Fig 4). Thus, edaravone treatment might provide a neuroprotective effect by reducing oxidative damage and it may contribute to limiting brain damage.

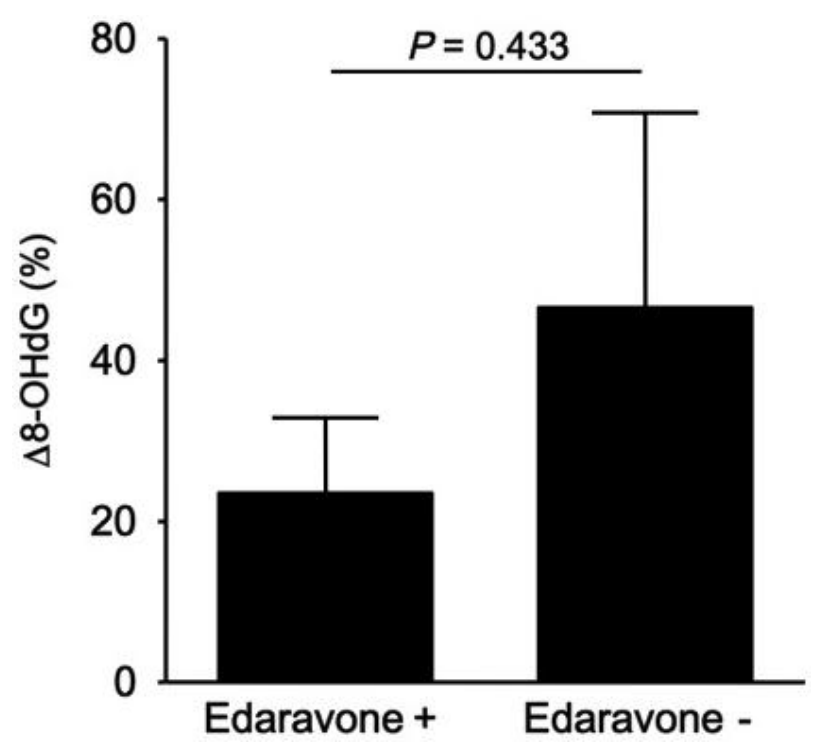

Fig. (4). Effect of edaravone on $\Delta 8-\mathrm{OHdG}$ in atherothrombotic infarction. $\Delta 8-\mathrm{OHdG}$ in patients who received edaravone tended to be reduced compared to those who did not receive edaravone. 
Table 3. Demographics and $\Delta 8$-OHdG Concentrations of Patients Treated with or Without Edaravone

\begin{tabular}{|c|c|c|c|}
\hline & Edaravone (+) & Edaravone (-) & $P$ value \\
\hline Male/female & $21 / 13$ & $5 / 5$ & 0.716 \\
\hline \multicolumn{4}{|l|}{ Stroke subtypes } \\
\hline \multicolumn{4}{|l|}{ Risk factors for arteriosclerosis } \\
\hline Hypertension, \% (n) & $56(19)$ & $80(8)$ & 0.271 \\
\hline Diabetes mellitus, \% (n) & $18(6)$ & $30(3)$ & 0.402 \\
\hline Hyperlipidemia, \% (n) & $44(15)$ & $10(1)$ & 0.067 \\
\hline Baseline NIHSS (range) & $11.1(2-37)$ & $6.1(1-9)$ & 0.842 \\
\hline NIHSS 14 days after admission (range) & $6.3(0-28)$ & $3(1-8)$ & 0.644 \\
\hline $\mathrm{mRS}$ at discharge & 2.5 & 2.7 & 0.597 \\
\hline \multicolumn{4}{|l|}{ Medications } \\
\hline Antithrombotic, \% (n) & $44(15)$ & $80(8)$ & 0.076 \\
\hline Glycerol, \% (n) & $86(29)$ & $60(6)$ & 0.175 \\
\hline Length of stay (days) & 32 & 29.7 & 0.123 \\
\hline
\end{tabular}

LAC; lacunar infarction, AT; atherothrombotic infarction, CE; cardioembolic infarction

\section{CONCLUSION}

The present study demonstrated a significant association between elevated urinary $8-\mathrm{OHdG}$ levels and acute ischemic stroke, especially for those with atherothrombotic infarctions. Because $\Delta 8-\mathrm{OHdG}$, the rate of change of urinary 8OHdG, showed a significant relationship to $\mathrm{mRS}$ scores at discharge, $\Delta 8$-OHdG may be a useful predictor of poor outcomes after ischemic stroke. Edaravone is useful for reducing oxidative damage, especially in patients with atherothrombotic infarctions, and thus, it may contribute to limiting brain damage in patients with ischemic stroke.

\section{COMPETING INTERESTS}

The authors declare that they have no competing interests.

\section{AUTHORS' CONTRIBUTIONS}

HN performed analyses, collected data and wrote the manuscript. KU helped to draft the manuscript and collected data. TI, HK, and FK helped to draft the manuscript. All authors read and approved the final manuscript.

\section{ACKNOWLEDGEMENT}

None declared.

\section{REFERENCES}

[1] El Kossi MM, Zakhary MM. Oxidative stress in the context of acute cerebrovascular stroke. Stroke 2000; 31: 1889-92.

[2] Madamanchi NR, Vendrov A, Runge MS. Oxidative stress and vascular disease. Arterioscler Thromb Vasc Biol 2005; 25: 29-38.

[3] Love S. Oxidative stress in brain ischemia. Brain Pathol 1999; 9; 119-31.

[4] Chamorro A. Role of inflammation in stroke and atherothrombosis Cerebrovasc Dis 2004; 17: 1-5.

[5] Zhang RL, Lu CZ, Ren HM, Xiao BG. Metabolic changes of arachidonic acid after cerebral ischemia-reperfusion in diabetic rats. Exp Neurol 2003; 184: 746-52.

[6] Hall ED, Braughler JM. Central nervous system trauma and stroke. II. Physiological and pharmacological evidence for involvement of oxygen radicals and lipid peroxidation. Free Radic Biol Med 1989; 6: 303-13.

[7] Halliwell B. Reactive oxygen species and the central nervous system. J Neurochem 1992; 59: 1609-23.

[8] Wu LL, Chiou CC, Chang PY, Wu JT. Urinary 8-OHdG: a marker of oxidative stress to DNA and a risk factor for cancer, atherosclerosis and diabetics. Clin Chim Acta 2004; 339: 1-9.

[9] Cooke MS, Lunec J, Evans MD. Progress in the analysis of urinary oxidative DNA damage. Free Radic Biol Med 2002; 33: 1601-14. 
[10] Cooke MS, Evans MD, Herbert KE, Lunec J. Urinary 8-oxo-2'deoxyguanosine--source, significance and supplements. Free Radic Res 2000; 32: 381-97.

[11] Zhang N, Komine-Kobayashi M, Tanaka R, Liu M, Mizuno Y, Urabe $\mathrm{T}$. Edaravone reduces early accumulation of oxidative products and sequential inflammatory responses after transient focal ischemia in mice brain. Stroke 2005; 36: 2220-5.

[12] Nishi H, Watanabe T, Sakurai H, Yuki S, Ishibashi A. Effect of MCI-186 on brain edema in rats. Stroke 1989; 20: 1236-40.

[13] Yamamoto T, Yuki S, Watanabe T, Mitsuka M, Saito KI, Kogure $\mathrm{K}$. Delayed neuronal death prevented by inhibition of increased hydroxyl radical formation in a transient cerebral ischemia. Brain Res 1997; 762: 240-2.

[14] The Edaravone Acute Brain Infarction Study Group: Effect of a novel free radical scavenger, edaravone (MCI-186), on acute brain infarction. Randomized, placebo-controlled, double-blind study at multicenters. Cerebrovasc Dis 2003; 15: 222-9.

[15] Goldstein LB, Samsa GP. Reliability of the national institutes of health stroke scale. Extension to non-neurologists in the context of a clinical trial. Stroke 1997; 28: 307-10.

[16] van Swieten JC, Koudstaal PJ, Visser MC, Schouten HJ, van Gijn J. Interobserver agreement for the assessment of handicap in stroke patients. Stroke 1988; 19: 604-7.

[17] National Institute of Neurological Disorders and Stroke: Classification of cerebrovascular disease III. Stroke 1990; 21: 637-76

[18] Saito S, Yamauchi H, Hasui Y, Kurashige J, Ochi H, Yoshida K. Quantitative determination of urinary 8-hydroxydeoxyguanosine (8-OHdG) by using ELISA. Res Commun Mol Pathol Pharmacol 2000; 107: 39-44.

[19] Taffi R, Nanetti L, Mazzanti L, et al. Plasma levels of nitric oxide and stroke outcome. J Neurol 2008; 255: 94-8.

[20] Kelly PJ, Morrow JD, Ning M, et al. Oxidative stress and matrix metalloproteinase-9 in acute ischemic stroke: the Biomarker Evaluation for Antioxidant Therapies in Stroke (BEAT-Stroke) study. Stroke 2008; 39: 100-4.

[21] Mizukoshi G, Katsura K, Katayama Y. Urinary 8-hydroxy-2'deoxyguanosine and serum S100beta in acute cardioembolic stroke patients. Neurol Res 2005; 27: 644-6.

[22] Uno M, Kitazato KT, Nishi K, Itabe H, Nagahiro S. Raised plasma oxidised LDL in acute cerebral infarction. J Neurol Neurosurg Psychiatry $2003 ; 74$ : 312-6.

[23] Musarrat J, Arezina-Wilson J, Wani AA. Prognostic and aetiological relevance of 8-hydroxyguanosine in human breast carcinogenesis. Eur J Cancer 1996; 32: 1209-14.
[24] Kojda G, Harrison D. Interactions between NO and reactive oxygen species: pathophysiological importance in atherosclerosis, hypertension, diabetes and heart failure. Cardiovasc Res 1999; 43: 562-71.

[25] Negishi H, Ikeda K, Kuga S, et al. The relation of oxidative DNA damage to hypertension and other cardiovascular risk factors in Tanzania. J Hypertens 2001; 19: 529-33.

[26] Martinet W, Knaapen MW, De Meyer GR, Herman AG, Kockx MM. Elevated levels of oxidative DNA damage and DNA repair enzymes in human atherosclerotic plaques. Circulation 2002; 106 : 927-32.

[27] Zhang W, Sato K, Hayashi T, et al. Extension of ischemic therapeutic time window by a free radical scavenger, Edaravone, reperfused with tPA in rat brain. Neurol Res 2004; 26: 342-8.

[28] Shinohara Y, Saito I, Kobayashi S, Uchiyama S. Edaravone (radical scavenger) versus sodium ozagrel (antiplatelet agent) in acute noncardioembolic ischemic stroke (EDO trial). Cerebrovasc Dis 2009; 27: 485-92.

[29] Shichinohe H, Kuroda S, Yasuda H, et al. Neuroprotective effects of the free radical scavenger Edaravone (MCI-186) in mice permanent focal brain ischemia. Brain Res 2004; 1029: 200-6.

[30] Toyoda K, Fujii K, Kamouchi M, et al. Free radical scavenger, edaravone, in stroke with internal carotid artery occlusion. J Neurol Sci 2004; 221: 11-7.

[31] Doi K, Suzuki Y, Nakao A, Fujita T, Noiri E. Radical scavenger edaravone developed for clinical use ameliorates ischemia/ reperfusion injury in rat kidney. Kidney Int 2004; 65: 1714-23.

[32] Lan J, Li W, Zhang F, et al. Inducible repair of oxidative DNA lesions in the rat brain after transient focal ischemia and reperfusion. J Cereb Blood Flow Metab 2003; 23: 1324-39.

[33] Uno M, Kitazato KT, Suzue A, et al. Inhibition of brain damage by edaravone, a free radical scavenger, can be monitored by plasma biomarkers that detect oxidative and astrocyte damage in patients with acute cerebral infarction. Free Radic Biol Med 2005; 39 ; 1109-16.

[34] Yagi K, Kitazato KT, Uno M, et al. Edaravone, a free radical scavenger, inhibits MMP-9-related brain hemorrhage in rats treated with tissue plasminogen activator. Stroke 2009; 40: 626-31.

[35] Yamashita T, Kamiya T, Deguchi K, et al. Dissociation and protection of the neurovascular unit after thrombolysis and reperfusion in ischemic rat brain. J Cereb Blood Flow Metab 2009; 29: 715-25. 